\title{
The Gauss2++ model: a comparison of different measure change specifications for a consistent risk neutral and real world calibration
}

\section{Christoph Berninger ${ }^{1,2} \oplus \cdot$ Julian Pfeiffer ${ }^{2}$}

Received: 1 July 2020 / Revised: 30 October 2020 / Accepted: 8 January 2021 /

Published online: 9 February 2021

(c) The Author(s) 2021

\begin{abstract}
Especially in the insurance industry interest rate models play a crucial role, e.g. to calculate the insurance company's liabilities, performance scenarios or risk measures. A prominant candidate is the 2-Additive-Factor Gaussian Model (Gauss2++ model)_in a different representation also known as the 2-Factor Hull-White model. In this paper, we propose a framework to estimate the model such that it can be applied under the risk neutral and the real world measure in a consistent manner. We first show that any time-dependent function can be used to specify the change of measure without loosing the analytic tractability of, e.g. zero-coupon bond prices in both worlds. We further propose two candidates, which are easy to calibrate: a step and a linear function. They represent two variants of our framework and distinguish between a short and a long term risk premium, which allows to regularize the interest rates in the long horizon. We apply both variants to historical data and show that they indeed produce realistic and much more stable long term interest rate forecast than the usage of a constant function, which is a popular choice in the industry. This stability over time would translate to performance scenarios of, e.g. interest rate sensitive fonds and risk measures.
\end{abstract}

Keywords 2-Factor Hull-White model $\cdot$ Gauss $2++$ model $\cdot$ Risk neutral and real world $\cdot$ Change of measure $\cdot$ Time-varying market price of risk

The original version of this article was revised: Equations under Section 3 and Section 3.3 are updated.

Christoph Berninger

christoph.berninger@stat.uni-muenchen.de

1 Department of Statistics, LMU München, Munich, Germany

2 ROKOCO GmbH, Ludwig-Ganghofer-Str. 6, 82031 Grünwald, Germany 


\section{Introduction}

Two prominent approaches to model the term structure of interest rates are the classes of equilibrium and no-arbitrage models. Most equilibrium models concentrate on the dynamic of the short-rate - the instantaneous interest rate-and derive interest rates with longer maturities from it. Prominent candidates of this model class include the models of Cox et al. [3], Duffie and Kan [9] and Vasicek [18]. No-arbitrage models focus on exactly fitting the term structure at a specific point in time to prevent arbitrage possibilities. Representatives of this class are introduced by Heath et al. [11] and Hull and White [12].

Applications of these models often relate to pricing interest rate derivatives, which is the reason why they are directly defined under the risk neutral measure most of the time. A general form of a one-factor short-rate model under the risk neutral measure is, e.g. given by

$$
d r(t)=\mu(t, r) d t+\sigma(t, r) d W(t),
$$

where $\mu$ and $\sigma$ are two functions, which can depend on time point $t$ and the short-rate $r$, and $W$ is a Brownian motion. A lot of advances in theoretic models and their estimation have been conducted in the last 30 years, but only in connection to pricing (see Diebold and Li [6]). Regarding these models little attention has been given to forecasting and risk management purposes (see Diebold and Li [6]). For these applications the corresponding model needs to be regarded under the real world measure. Under this measure the corresponding one factor short-rate model has the following dynamic

$$
d r(t)=[\mu(t, r)+\lambda(t, r) \sigma(t, r)] d t+\sigma(t, r) d \widetilde{W}(t),
$$

where $\lambda$ is the market price of risk and can also depend on $t$ and $r$. $\widetilde{W}$ is a Brownian motion under the real world measure. The exact functional choice for $\lambda$ completes the model specification under the real world measure. Dai and Singleton [5] as well as Jong [14] use a fixed multiple of the model's variance for the market price of risk and investigate the in sample fit of specific short-rate models, but do not focus on forecasting. Duffee [8] concludes that the class of term structure models analysed in Dai and Singleton [5] fail in forecasting. He argues that a restriction for the market price of risk to be a fixed multiple of the variance reduces the flexibility of the model. Hull et al. [13] stress that the market price of risk for a model with few factors should be time-dependent. This results not from an economic interpretation but from a modelling issue because of an insufficient number of factors (see Hull et al. [13]). They estimated the market price of risk based on historical 3-month and 6-month interest rates and came to a similar result as Ahmad and Wilmott [1], Cox and Pedersen [4] and Stanton [17]. But they argue that this value is only valid in the short horizon. Keeping this market price of risk constant could lead to extreme risk premiums and interest rates in the long horizon.

In this paper we tackle exactly this problem for the Gauss $2++$ model. Instead of assuming a constant, we assume a time-varying function for the market price of risk. 
In contrast to Hull et al. [13], who estimate the market price of risk for each forecasting horizon individually, we propose two parametric functions. The step function is the easiest non-constant function, which allows to model a market price of risk valid in the short and one valid in the long horizon. The linear function assumes that the market price of risk in the short horizon converges linearly to a long-term level. With these simplified time-dependent functions it is possible to account for the problem mentioned by Hull et al. [13] and the functions can still be easily estimated by historical data or calibrated in a forward looking manner to interest rate forecasts.

In our backtest we use a very similar calibration approach as described in Korn and Wagner [15]. The framework illustrated in this monograph has been developed by the Fraunhofer ITWM on behalf of the Produktinformationsstelle Altersvorsorge $\mathrm{GmbH}$ (PIA) and is the industry standard to classify packaged retail and insurance based investment products (PRIIPs) into chance-risk classes. For the interest rate model they use a Gauss2++ model with a presumed constant market price of risk. Following their calibration procedure allows us to compare our results to real applications in the insurance industry.

The structure of the paper is as follows. In Sect. 2 we introduce the Gauss2++ model under the risk neutral and the real world measure in a very general framework. In Sect. 3 we propose the constant function for comparison reason as well as the step and the linear function to specify the change of measure and explain how they can be estimated. All three variants of the Gauss2++ model are applied to data and backtested for the last 3 years in Sect. 4 . In the final section the results are summarized and concluded.

\section{The Gauss2++ model in the risk neutral and the real world}

Throughout this section a filtered probability space $\left(\Omega, \mathcal{F},\left(\mathcal{F}_{t}\right)_{t \in[0, \mathcal{T}]}, \mathbb{M}\right)$ is given, where $\mathbb{M}$ is either the risk neutral measure $\mathbb{Q}$ with respect to the bank account or the real world measure $\mathbb{P}$. $\mathcal{T}$ represents an appropriate modelling horizon. The bank account $(B(t))_{t \in[0, \mathcal{T}]}$ is given by

$$
d B(t)=r(t) B(t) d t, \quad B(0)=1,
$$

where $r(t)$ denotes the short-rate. We further adopted notations and descriptions of the Gauss2++ model from the relevant chapters in Brigo and Mercurio [2].

\subsection{The Gauss2++ model under the risk neutral measure}

Short-rate models differ in the underlying process for the short-rate. The Gauss $2++$ model assumes that the short-rate is given by a sum of two correlated normally distributed processes, $(x(t))_{t \in[0, \mathcal{T}]}$ and $(y(t))_{t \in[0, \mathcal{T}}$, and a deterministic function $\varphi$, which is well defined on the time interval $[0, \mathcal{T}]$ :

$$
r(t)=x(t)+y(t)+\varphi(t), \quad r(0)=r_{0},
$$


where $r_{0}$ is the short-rate at time point 0 . The processes $(x(t))_{t \in[0, \mathcal{T}]}$ and $(y(t))_{t \in[0, \mathcal{T}]}$ satisfy under the risk neutral measure $\mathbb{Q}$ the following stochastic differential equations

$$
\begin{aligned}
d x(t) & =-a x(t) d t+\sigma d W^{1}(t), & & x(0)=0, \\
d y(t) & =-b y(t) d t+\eta d W^{2}(t), & & y(0)=0, \\
\rho d t & =d W^{1}(t) d W^{2}(t), & &
\end{aligned}
$$

where $a, b, \sigma, \eta$ are non-negative constants and $-1 \leq \rho \leq 1$ is the instantaneous correlation between the two Brownian motions $W^{1}$ and $W^{2}$.

Short-rate models derive spot rates via prices of zero-coupon bonds. As the short-rate in the Gauss $2++$ model is normally distributed, there exists an analytic solution for a zero-coupon bond price, $P(t, T)$, at time point $t$ and maturity $T$ :

$$
P(t, T)=e^{-\int_{t}^{T} \varphi(s) d s-B(a, t, T) x(t)-B(b, t, T) y(t)+\frac{1}{2} V(t, T)},
$$

where

$$
B(z, t, T)=\frac{1-e^{-z(T-t)}}{z}
$$

and

$$
\begin{aligned}
V(t, T)= & \frac{\sigma^{2}}{a^{2}}\left[(T-t)+\frac{2}{a} e^{-a(T-t)}-\frac{1}{2 a} e^{-2 a(T-t)}-\frac{3}{2 a}\right] \\
& +\frac{\eta^{2}}{b^{2}}\left[(T-t)+\frac{2}{b} e^{-b(T-t)}-\frac{1}{2 b} e^{-2 b(T-t)}-\frac{3}{2 b}\right] \\
& +2 \rho \frac{\sigma \eta}{a b}\left[(T-t)+\frac{e^{-a(T-t)}-1}{a}+\frac{e^{-b(T-t)}-1}{b}-\frac{e^{-(a+b)(T-t)}-1}{a+b}\right] .
\end{aligned}
$$

A derivation can be found in Brigo and Mercurio [2]. With formula (1) for the zerocoupon bond price under the risk neutral measure spot rates can be directly derived via

$$
r(t, T)=\frac{-\ln (P(t, T))}{T-t}
$$

where $r(t, T)$ represents the spot rate at time point $t$ and a maturity of $T$.

The financial market we actually model consists of a bank account and a set of zero-coupon bonds, $P(t, T)$, which differ in the maturity $T$. The dynamic of a zero-coupon bond price can be derived from the bond price formula in (1) by applying Ito's formula and is given by

$$
d P(t, T)=P(t, T)\left[r(t) d t-\sigma B(a, t, T) d W^{1}(t)-\eta B(b, t, T) d W^{2}(t)\right] .
$$

A detailed derivation can be found in "Appendix 1". Note that all assets have the same drift as it is the case in the risk neutral world. 


\subsection{The Gauss2++ model under the real world measure}

To calculate performance scenarios and risk indicators the Gauss $2++$ model must be regarded under the real world measure $\mathbb{P}$.

\subsubsection{The change of measure}

By specifying the Gauss $2++$ model under the risk neutral measure, we implicitly assume an arbitrage free market. Therefore, we can make the transition to a real world measure $\mathbb{P}$ by defining the change of measure according to Girsanov, who states that a progressive and square-integrable process $\boldsymbol{\Phi}=\left(\Phi^{1}(t), \Phi^{2}(t), \ldots, \Phi^{d}(t)\right)_{t \in[0, \mathcal{T}]}$ determines a new probability measure $\mathbb{P}$ such that if $(\widehat{\boldsymbol{W}}(t))_{t \in[0, \mathcal{T}}$ is a standard $d$-dimensional $\left(\mathcal{F}_{t}\right)_{t \in[0, \mathcal{T}]}$-Brownian motion under $\mathbb{Q}$, then

$$
\breve{\boldsymbol{W}}(t):=\widehat{\boldsymbol{W}}(t)+\int_{0}^{t} \boldsymbol{\Phi}(s) d s
$$

defines a standard $d$-dimensional $\left(\mathcal{F}_{t}\right)_{t \in[0, \mathcal{T}}$-Brownian motion under $\mathbb{P}$ (see Girsanov [10]).

The Gauss $2++$ model is a two-factor model and $\boldsymbol{\Phi}$ is therefore two-dimensional. Its components can be interpreted as the market price of risk for each factor in the model. We will represent $\boldsymbol{\Phi}$ such that the resulting processes $(x(t))_{t \in[0, \mathcal{T}]}$ and $(y(t))_{t \in[0, \mathcal{T}]}$ still belong to the class of Ornstein-Uhlenbeck processes under $\mathbb{P}$

$$
\boldsymbol{\Phi}(t)=\left(\begin{array}{c}
\Phi^{1}(t) \\
\Phi^{2}(t)
\end{array}\right)=\left(\begin{array}{c}
-\frac{a d_{x}(t)}{\sigma} \\
-\frac{b d_{y}(t)}{\eta \sqrt{1-\rho^{2}}}+\frac{\rho a d_{x}(t)}{\sigma \sqrt{1-\rho^{2}}}
\end{array}\right) .
$$

Note that we restrict $\boldsymbol{\Phi}$ to be a function of time. By this the change of measure only changes the mean reversion level. More general measure change specification can be applied. For example, Diez and Korn [7] introduce a measure change for the 1-Factor Vasicek model, which influences the mean reversion level as well as the mean reversion speed.

The conditions for the Girsanov theorem translate directly to the functions $d_{x}(t)$ and $d_{y}(t)$. In the following we will specify the change of measure via $d_{x}(t)$ and $d_{y}(t)$. An appropriate interpretation of these functions will be given in Sect. 2.2.2.

\subsubsection{The dynamics under the real world measure $\mathbb{P}$}

With the representation of $\boldsymbol{\Phi}$ as in (3) the dynamics of the processes $x$ and $y$ in the Gauss $2++$ model change according to Girsanov to

$$
\begin{array}{ll}
d x(t)=a\left(d_{x}(t)-x(t)\right) d t+\sigma d \tilde{W}^{1}(t), & x(0)=0, \\
d y(t)=b\left(d_{y}(t)-y(t)\right) d t+\eta d \tilde{W}^{2}(t), & y(0)=0,
\end{array}
$$


where $\widetilde{W}^{1}$ and $\widetilde{W}^{2}$ are two correlated Brownian motions under $\mathbb{P}$. The derivation can be found in "Appendix 2". We observe that $x$ and $y$ are still Ornstein-Uhlenbeck processes with the solutions

$$
\begin{aligned}
& x(t)=\int_{0}^{t} e^{-a(t-u)} a d_{x}(u) d u+\sigma \int_{0}^{t} e^{-a(t-u)} d \widetilde{W}(u), \\
& y(t)=\int_{0}^{t} e^{-b(t-u)} b d_{y}(u) d u+\eta \int_{0}^{t} e^{-b(t-u)} d \widetilde{W}(u) .
\end{aligned}
$$

The mean reversion level of each process at time point $t$ amounts to $d_{x}(t)$ and $d_{y}(t)$, respectively. Recall that the sum of $x(t)$ and $y(t)$ and a deterministic function $\varphi(t)$ under the risk neutral measure adds up to the instantaneous return rate $r(t)$ of a risk free investment. Changing the measure changes the mean reversion level at time point $t$ from 0 to $d_{x}(t)$ for the process $x$ and to $d_{y}(t)$ for the process $y$. Therefore, $d_{x}(t)+d_{y}(t)$ can be interpreted as the local long run risk premium of the short-ratethe amount, which is added in the real world to the risk neutral short-rate in the long run, if $d_{x}(t)+d_{y}(t)$ would stay constant over time. If this amount is negative, future bond prices increase in expectation compared to the risk neutral world and a risk averse investor, therefore, gets compensated for the risk of investing in a risky bond. This means in contrast to equity prices, in a market where investors are risk averse, future interest rates tend to be lower in the real world than in the risk neutral world (see, e.g. Hull et al. [13]). Therefore, $d_{x}(t)$ and $d_{y}(t)$ can be interpreted as the local long run risk premium the corresponding risk factor is mean reverting to at time point $t$.

In the following we will specify the change of measure by these two functions instead of the market prices of risk. The market price of risk of each risk factor is then directly defined by these two functions.

$$
\begin{aligned}
& \text { Market price of risk of risk factor 1: }-\frac{a d_{x}(t)}{\sigma} \\
& \text { Market price of risk of risk factor 2: }-\frac{b d_{y}(t)}{\eta \sqrt{1-\rho^{2}}}+\frac{\rho a d_{x}(t)}{\sigma \sqrt{1-\rho^{2}}} .
\end{aligned}
$$

If we assume a step or a piecewise linear function for $d_{x}(t)$ and $d_{y}(t)$ the functional form of the individual market prices of risk are the same.

The dynamics of a zero-coupon bond with maturity $T$ under $\mathbb{P}$ has the following form

$$
\begin{aligned}
d P(t, T)= & P(t, T)\left[r(t)-B(a, t, T) a d_{x}(t)-B(b, t, T) b d_{y}(t)\right] d t \\
& -P(t, T) B(a, t, T) \sigma d \widetilde{W}^{1}(t)-P(t, T) B(b, t, T) \eta d \widetilde{W}^{2}(t)
\end{aligned}
$$

The derivation can be found in "Appendix 3". 


\subsubsection{The bond price formula under the real world measure}

The price of a zero-coupon bond under $\mathbb{P}$ is obtained by the same analytic formula as in (1). The only difference is that the $x$ - and the $y$-process are now regarded under the real world measure (see Diez and Korn [7]). In the following we will shortly explain why the formula does not change under this new measure.

To calculate the price of a zero-coupon bond under the real world measure we use the following conditional expectation

$$
\frac{P(t, T)}{X_{P(t, T)}(t)}=E^{\mathbb{P}}\left[\frac{P(T, T)}{X_{P(t, T)}(T)} \mid \mathcal{F}_{t}\right],
$$

where $X_{P(t, T)}$ represents the cash flow, with which we have to discount the zerocoupon bond such that the discounted price process is a martingale under $\mathbb{P}$. The dynamic of $X_{P(t, T)}$ coincides with the deterministic part of the zero-coupon bond price dynamic in (8) and is therefore specified by the change of measure:

$$
d X_{P(t, T)}(t)=X_{P(t, T)}(t)\left[r(t)-B(a, t, T) a d_{x}(t)-B(b, t, T) b d_{y}(t)\right] d t, \quad X_{P(t, T)}(0)=1 .
$$

A short proof can be found in "Appendix 4". The solution of this dynamic is given by

$$
X_{P(t, T)}(t)=e^{\int_{0}^{t}\left(r(u)-B(a, u, T) a d_{x}(u)-B(b, u, T) b d_{y}(u)\right) d u} .
$$

The price of a zero-coupon bond at time point $t$ is therefore given by

$$
P(t, T)=E^{\mathbb{P}}\left[\frac{X_{P(t, T)}(t)}{X_{P(t, T)}(T)} \mid \mathcal{F}_{t}\right] .
$$

The ratio in the expectation amounts to

$$
\frac{X_{P(t, T)}(t)}{X_{P(t, T)}(T)}=e^{-\int_{t}^{T}\left(r(u)-B(a, u, T) a d_{x}(u)-B(b, u, T) b d_{y}(u)\right) d u} .
$$

To determine the distribution of this ratio, we first derive the distribution of the integral in the exponent, i.e.

$$
I(t, T):=\int_{t}^{T}\left(r(u)-B(a, u, T) a d_{x}(u)-B(b, u, T) b d_{y}(u)\right) d u .
$$

It can be shown that $I(t, T)$ is normally distributed with mean

$$
M(t, T)=\int_{t}^{T} \varphi(u) d u+\frac{1-e^{-a(T-t)}}{a} x(t)+\frac{1-e^{-b(T-t)}}{b} y(t)
$$

and variance 


$$
\begin{aligned}
V(t, T)= & \frac{\sigma^{2}}{a^{2}}\left[(T-t)+\frac{2}{a} e^{-a(T-t)}-\frac{1}{2 a} e^{-2 a(T-t)}-\frac{3}{2 a}\right] \\
& +\frac{\eta^{2}}{b^{2}}\left[(T-t)+\frac{2}{b} e^{-b(T-t)}-\frac{1}{2 b} e^{-2 b(T-t)}-\frac{3}{2 b}\right] \\
& +2 \rho \frac{\sigma \eta}{a b}\left[(T-t)+\frac{e^{-a(T-t)}-1}{a}+\frac{e^{-b(T-t)}-1}{b}-\frac{e^{-(a+b)(T-t)}-1}{a+b}\right] .
\end{aligned}
$$

The variance is the same as in the risk neutral world as the change of measure does not influence the variance of the processes. Note that also the mean has the same form as in the risk neutral case as the terms $B(a, u, T) a d_{x}(u)$ and $B(b, u, T) b d_{y}(u)$ in $I(t, T)$ cancel out in the calculations. The derivations can be found in "Appendix 5".

The expression $e^{-I(t, T)}$ is therefore log-normally distributed and the zero-coupon bond price under $\mathbb{P}$ is given by the same analytic formula as under $\mathbb{Q}$ :

$$
\begin{aligned}
P(t, T) & =E^{\mathbb{P}}\left[e^{-\int_{t}^{T} r(u)-B(a, u, T) a d_{x}(u)-B(b, u, T) b d_{y}(u) d u} \mid \mathcal{F}_{t}\right] \\
& =e^{-M(t, T)+\frac{1}{2} V(t, T)} \\
& =e^{-\int_{t}^{T} \varphi(u) d u-\frac{1-e^{-a(T-t)}}{a} x(t)-\frac{1-e^{-b(T-t)}}{b} y(t)+\frac{1}{2} V(t, T)} .
\end{aligned}
$$

\section{Local long run risk premium functions-specification and calibration}

In the following three different types of functions for $d_{x}(t)$ and $d_{y}(t)$ are introduced: the constant, the step and the linear function. Following the interpretation in Sect. 2.2.2 these functions represent the long run risk premium for each risk factor at a specific time point $t$ in the Gauss $2++$ model. The functional equations of the three types are

$$
\begin{aligned}
\text { Constant: } & d_{x}(t)=d_{x} \\
& d_{y}(t)=d_{y} \\
\text { Step: } & d_{x}(t)=\mathbb{1}_{t \leq \tau} d_{x}+\mathbb{1}_{t>\tau} l_{x} \\
& d_{y}(t)=\mathbb{1}_{t \leq \tau} d_{y}+\mathbb{1}_{t>\tau} l_{y} \\
\text { Linear: } & d_{x}(t)=\mathbb{1}_{t \leq \tau}\left(1-m_{x} t\right) d_{x}+\mathbb{1}_{t>\tau} l_{x} \\
& d_{y}(t)=\mathbb{1}_{t \leq \tau}\left(1-m_{y} t\right) d_{y}+\mathbb{1}_{t>\tau} l_{y}
\end{aligned}
$$

where $d_{x}, l_{x}, m_{x}$ and $d_{y}, l_{y}, m_{y}$ are real valued constants and $\mathbb{1}_{A}$ represents the indicator function of a subset $A$.

The constant function assumes that the local long run risk premium is constant for the whole modelling horizon. The latter two functions distinguish between a local long run risk premium valid in the short and in the long horizon, seperated at time point $\tau$. As mentioned in Sect. 2.2.2 the same holds for the market price of risk, respectively. Hull et al. [13] argue that a time-varying market price of risk is 
necessary to account for unobserved risk factors and to prevent unrealistic interest rate forecasts in the long horizon. They therefore estimate an individual market price of risk for each forecasting horizon. We use a more parsimonious function with regard to the number of parameters. The step function we propose is the simplest time-varying function that expects that the local long run risk premium differs in the short and the long horizon but is still constant in each period. The linear function implements the property that the local long run risk premium in the short horizon approaches the long term level linearly. The simplicity of these functions allows a straight forward calibration to interest rate forecasts.

Because of the distributional properties of the Gauss $2++$ model the expected values for interest rates under the real world measure $\mathbb{P}$ for any future time point can be calculated:

$$
E^{\mathbb{P}}[r(t, T)]=E^{\mathbb{Q}}[r(t, T)]+\frac{B(a, t, T)}{T-t} R P_{x}(t)+\frac{B(b, t, T)}{T-t} R P_{y}(t),
$$

where $R P_{x}(t)$ and $R P_{y}(t)$ represent the actual risk premium of the short-rate at time point $t$ for each risk factor and are given by the first integral in (6) and (7)

$$
\begin{aligned}
& R P_{x}(t):=\int_{0}^{t} e^{-a(t-u)} a d_{x}(u) d u, \\
& R P_{y}(t):=\int_{0}^{t} e^{-b(t-u)} b d_{y}(u) d u .
\end{aligned}
$$

For the constant, the step and the linear function these integrals can be easily calculated. To get the risk premium for longer maturities the functions $R P_{x}(t)$ and $R P_{y}(t)$ are weighted by a loading function, which accounts for the different riskiness of the corresponding zero-coupon bonds

$$
\frac{B(a, t, T)}{T-t} \quad \text { and } \quad \frac{B(b, t, T)}{T-t} .
$$

To calibrate the local long run risk premium functions, $d_{x}(t)$ and $d_{y}(t)$, the parameters of the functions are chosen in such a way that the model meets specific interest rate forecasts in expectation. For the constant type two interest rate forecasts are needed. For the other two types four interest rate forecasts are necessary-two short term and two long term forecasts. The time parameter $\tau$, which determines the separation between the short and the long term local long run risk premium must lie between the forecasting horizons of the two short and the two long term forecasts.

In Fig. 1 the three types of local long run risk premium functions have been exemplary calibrated. $\tau$ has been set to 24 months, which is the forecasting horizon of the short term interest rate forecasts.

In the following subsections the calibration procedures for all three types of local long run risk premium functions, which are applied in this paper, are described. 


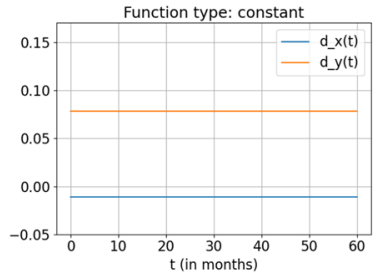

(a)

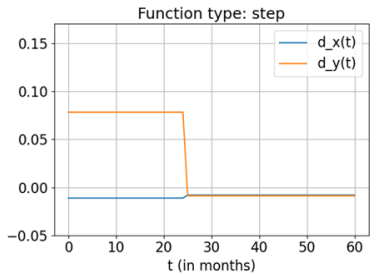

(b)

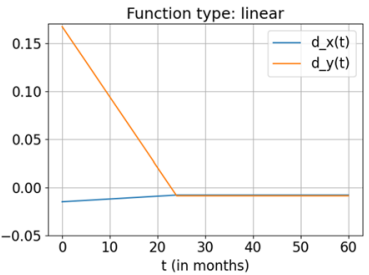

(c)

Fig. 1 Local long run risk premium functions

\subsection{The constant function}

The constant functions represented in Fig. 1a implement a constant local long run risk premium for the whole modelling horizon, which can amount to 40 years or more for actual applications in the insurance industry, e.g. to classify certified pension contracts into risk classes. The absolute risk premiums, $R P_{x}(t)$ and $R P_{y}(t)$, are given by:

$$
\begin{aligned}
& R P_{x}(t)=\left(1-e^{-a t}\right) d_{x}, \\
& R P_{y}(t)=\left(1-e^{-b t}\right) d_{y} .
\end{aligned}
$$

Note that if $t \rightarrow \infty, R P_{x}(t)$ and $R P_{y}(t)$ indeed converge to $d_{x}$ and $d_{y}$, the long run risk premiums, respectively. To calibrate the parameters of the constant functions two interest rate forecasts, $\hat{r}\left(t_{1}, T_{1}\right)$ and $\hat{r}\left(t_{2}, T_{2}\right)$, are used. Plugging the absolute risk premium functions, $R P_{x}(t)$ and $R P_{y}(t)$, into (11) and setting the expectations equal to the interest rate forecasts results in the following two equations

$$
\begin{aligned}
& \text { (I) } \hat{r}\left(t_{1}, T_{1}\right) \stackrel{!}{=} E^{Q}\left[r\left(t_{1}, T_{1}\right)\right]+\frac{B\left(a, t_{1}, T_{1}\right)}{\left(T_{1}-t_{1}\right)}\left(1-e^{-a t_{1}}\right) d_{x}+\frac{B\left(b, t_{1}, T_{1}\right)}{\left(T_{1}-t_{1}\right)}\left(1-e^{-b t_{1}}\right) d_{y}, \\
& \text { (II) } \hat{r}\left(t_{2}, T_{2}\right) \stackrel{!}{=} E^{Q}\left[r\left(t_{2}, T_{2}\right)\right]+\frac{B\left(a, t_{2}, T_{2}\right)}{\left(T_{2}-t_{2}\right)}\left(1-e^{-a t_{2}}\right) d_{x}+\frac{B\left(b, t_{2}, T_{2}\right)}{\left(T_{2}-t_{2}\right)}\left(1-e^{-b t_{2}}\right) d_{y} .
\end{aligned}
$$

As the expectations are linear functions in $d_{x}$ and $d_{y}$, the two parameters can be easily determined.

The constant function for the local long run risk premium in the Gauss2++ model and this calibration procedure is a standard approach in the insurance industry. As the values for $d_{x}$ and $d_{y}$ determine the risk premium for the whole modelling horizon, their calibration is crucial for the model's interest rate distribution. Especially if the interest rate forecasts used for the calibration have a short forecasting horizon, the resulting distribution in the long horizon is very sensitive to these forecasts. For example if the interest rate forecasts and the forward rates-calculated from the current yield curve-are very different, to reach the forecasts a huge risk premium is necessary, which might be valid in the short horizon, but produces extreme interest rates in the long horizon. The next two functions account for this problem by representing a time-varying local long run risk premium. 


\subsection{The step function}

The step functions represented in Fig. $1 \mathrm{~b}$ take the same value as the corresponding constant function up to time $\tau$ as the same interest rate forecasts have been used for the short horizon, but then they jump to a different level to account for the risk premium in the long horizon. Similar to the constant function the absolute risk premium functions can easily be calculated and amount to

$$
\begin{aligned}
& R P_{x}(t)=\left(e^{-a(t-\min (t, \tau))}-e^{-a t}\right) d_{x}+\left(1-e^{-a(t-\min (t, \tau))}\right) l_{x}, \\
& R P_{y}(t)=\left(e^{-b(t-\min (t, \tau))}-e^{-b t}\right) d_{y}+\left(1-e^{-b(t-\min (t, \tau))}\right) l_{y} .
\end{aligned}
$$

Note that if $t \rightarrow \infty, R P_{x}(t)$ and $R P_{y}(t)$ now converge to $l_{x}$ and $l_{y}$, respectively. To calibrate the four parameters of the step function two short term and two long term interest rate forecasts are used resulting in the following equations:

$$
\begin{aligned}
& \text { (I) } \hat{r}\left(t_{1}, T_{1}\right) \stackrel{!}{=} E^{Q}\left[r\left(t_{1}, T_{1}\right)\right]+\frac{B\left(a, t_{1}, T_{1}\right)}{\left(T_{1}-t_{1}\right)} R P_{x}\left(t_{1}\right)+\frac{B\left(b, t_{1}, T_{1}\right)}{\left(T_{1}, t_{1}\right)} R P_{y}\left(t_{1}\right), \\
& \text { (II) } \hat{r}\left(t_{2}, T_{2}\right) \stackrel{!}{=} E^{Q}\left[r\left(t_{2}, T_{2}\right)\right]+\frac{B\left(a, t_{2}, T_{2}\right)}{\left(T_{2}-t_{2}\right)} R P_{x}\left(t_{2}\right)+\frac{B\left(b, t_{2}, T_{2}\right)}{\left(T_{2}-t_{2}\right)} R P_{y}\left(t_{2}\right), \\
& \text { (III) } \hat{r}\left(t_{3}, T_{3}\right) \stackrel{!}{=} E^{Q}\left[r\left(t_{3}, T_{3}\right)\right]+\frac{B\left(a, t_{3}, T_{3}\right)}{\left(T_{3}-t_{3}\right)} R P_{x}\left(t_{3}\right)+\frac{B\left(b b t_{3}, T_{3}\right)}{\left(T_{3}-t_{3}\right)} R P_{y}\left(t_{3}\right), \\
& \text { (IV) } \hat{r}\left(t_{4}, T_{4}\right) \stackrel{!}{=} E^{Q}\left[r\left(t_{4}, T_{4}\right)\right]+\frac{B\left(a, t_{4}, T_{4}\right)}{\left(T_{4}-t_{4}\right)} R P_{x}\left(t_{4}\right)+\frac{B\left(b, t_{4}, T_{4}\right)}{\left(T_{4}-t_{4}\right)} R P_{y}\left(t_{4}\right),
\end{aligned}
$$

where $t_{1} \leq t_{2}<t_{3} \leq t_{4}$. $\tau$ must lie between $t_{2}$ and $t_{3}$, i.e. $t_{2} \leq \tau<t_{3}$.

Instead of interest rate forecasts direct forecasts of the absolute risk premium of the short-rate can be used. This approach is applied by Hull et al. [13], who estimate risk premiums for each forecasting horizon from historical data, but they also scale their result to a long term short-rate forecast. Another possible approach is to take the ultimate forward rate (UFR) from Solvency II as a long term target, which is reached at a future time point with a specific percentage (e.g. $95 \%$ of the UFR in 40 years) and to $100 \%$ in the limit, i.e. $t \rightarrow \infty$.

\subsection{The linear function}

The linear functions represented in Fig. 1c avoid the sudden jump as it is the case in the step functions and converge in the short term linearly to a long term level. The absolute risk premiums at time point $t$ can be calculated as before and amount to

$$
\begin{aligned}
R P_{x}(t) & =\left(\left(e^{-a(t-\min (t, \tau))}-e^{-a t}\right)\left(1+\frac{m_{x}}{a}\right)-e^{-a(t-\min (t, \tau))} m_{x} \min (t, \tau)\right) d_{x} \\
& +\left(1-e^{-a(t-\min (t, \tau))}\right) l_{x}, \\
R P_{y}(t) & =\left(\left(e^{-b(t-\min (t, \tau))}-e^{-b t}\right)\left(1+\frac{m_{y}}{b}\right)-e^{-b(t-\min (t, \tau))} m_{y} \min (t, \tau)\right) d_{y} \\
& +\left(1-e^{-b(t-\min (t, \tau))}\right) l_{y} .
\end{aligned}
$$


Note again that if $t \rightarrow \infty, R P_{x}(t)$ and $R P_{y}(t)$ converge to $l_{x}$ and $l_{y}$, the long term risk premiums, respectively. To calibrate $d_{x}, l_{x}, d_{y}$ and $l_{y}$ four interest rate forecasts as for the step function are used. By imposing that the absolute risk premium functions, $R P_{x}(t)$ and $R P_{y}(t)$, are differentiable at the forecasting horizon $\tau$ to prevent a kink in the absolute risk premium function, two further conditions are incorporated to specify $m_{x}$ and $m_{y}$ :

$$
\begin{aligned}
&(\mathrm{V})\left(R P_{x}\right)_{-}^{\prime}(\tau)=\left(R P_{x}\right)_{+}^{\prime}(\tau), \\
& \text { (VI) }\left(R P_{y}\right)_{-}^{\prime}(\tau)=\left(R P_{y}\right)_{+}^{\prime}(\tau),
\end{aligned}
$$

where $(R P .)_{-}^{\prime}(\tau)$ and $(R P .)_{+}^{\prime}(\tau)$ denote the derivative from the left and from the right, respectively. Solving the equations for $m_{x}$ and $m_{y}$ leads to the following closed form solutions reducing the number of free parameters to four:

$$
\begin{aligned}
& m_{x}=\frac{d_{x}-l_{x}}{d_{x} \tau}, \\
& m_{y}=\frac{d_{y}-l_{y}}{d_{y} \tau} .
\end{aligned}
$$

Note that with this condition the same number of interest rate forecasts as for the step function are needed to calibrate $d_{x}(t)$ and $d_{y}(t)$.

\section{Results}

In this section the calibration results of three variants of our framework for the Gauss $2++$ model are presented. The variants differ in the assumption about the local long run risk premium functions, which determine the change from the risk neutral to the real world measure. Variant 1 assumes a constant, variant 2 a step and variant 3 a linear local long run risk premium function for the risk factors. In the first subsection the three variants of the Gauss $2++$ model are compared if calibrated at the same valuation date. In Sect. 4.2 we show with a backtest over the last three years that variant 2 and 3 produce much more stable interest rate scenarios for the long forecasting horizon over this time period. This stability would transfer to performance scenarios and risk measures of, e.g. an interest rate sensitive fonds.

\subsection{Calibration at one valuation date}

The calibration process of the Gauss $2++$ model can be split into two steps. In the first step the model is calibrated under the risk neutral measure. This step does not depend on the choice of the local long run risk premium function and is therefore the same for all modelling cases. In the second step the change of measure is calibrated. The choice of the local long run risk premium function plays an important role and leads to different interest rate scenarios, performance measures and risk indicators. 
Table 1 Parameters of the Gauss $2++$ model calibrated at 31.12.2019

\begin{tabular}{lllll}
\hline$a$ & $b$ & $\sigma$ & $\eta$ & $\rho$ \\
\hline 0.2997 & 0.0407 & 0.0114 & 0.0114 & -0.9998 \\
\hline
\end{tabular}

To calibrate the model at a specific valuation date under the risk neutral measure the term structure of interest rate swaps and swaption volatilities at this date are used. In the Gauss $2++$ model the deterministic and time-dependent function $\varphi$ ensures the market consistency regarding the current term structure by being defined as follows:

$$
\varphi(t)=f^{M}(0, t)+\frac{\sigma^{2}}{2 a}\left(1-e^{-a t}\right)^{2}+\frac{\eta^{2}}{2 b}\left(1-e^{-b t}\right)^{2}+\rho \frac{\sigma \eta}{a b}\left(1-e^{-a t}\right)\left(1-e^{b t}\right) .
$$

$f^{M}(0, t)$ represents the instantaneous forward rate at time point 0 for a maturity $t$, i.e. $f^{M}(0, t)=\frac{\partial P^{M}(0, t)}{\partial T}$, where $\frac{\partial P^{M}}{\partial T}$ denotes the partial derivative with respect to the second argument and $P^{M}(0, t)$ is the market zero-coupon bond price. For the derivation and further information the reader is referred to Brigo and Mercurio [2]. The parameters $a, b, \sigma, \eta$ and $\rho$ of the model are chosen in such a way that the model prices of the considered swaptions coincide with the market prices. For this the downhill simplex algorithm ${ }^{1}$ is used to minimize the root mean squared error (RMSE):

$$
R M S E=\sqrt{\sum_{i=1}^{N}\left(C_{\text {Model, } i}(a, b, \sigma, \eta, \rho)-C_{\text {Market }, i}\right)^{2}},
$$

where $C_{\text {Model, } i}$ represents the model price of swaption $i$ of the Gauss2++ model and $C_{\text {Market, } i}$ is the market price of that swaption. The swaptions considered in the calibration process differ with respect to their tenor and maturity combination, which is denoted by the subscript $i$. $N$ represents the number of considered swaptions. The analytic formula for the price of a swaption in the Gauss $2++$ model can be found in Brigo and Mercurio [2]. Table 1 shows the result of a calibration at the 31.12.2019. We used at-the-money receiver swaptions with a maturity and tenor combination of $\{5,7,10,12,15,20\} \times\{5,7,10,12,15,20\}$, i.e. in total $N=36$ swaption prices. The $R M S E$ amounts to 0.0619 . In the optimization we further restricted $\rho$ to lie between -1 and 1 as well as all other parameters to be $>0$.

These parameters together with the current interest rate curve determine the dynamics of the Gauss2++ model under the risk neutral measure.

In the second step the local long run risk premium functions, which determine the change of measure, are calibrated to interest rate forecasts as described in Sects. 3.1-3.3. For the short term interest rate forecasts we use forecasts published by the OECD for a 3-month and a 10-year interest rate. To take the OECD forecasts

\footnotetext{
${ }^{1}$ For a detailed description of this algorithm - also known as the Nelder-Mead algorithm — the reader is referred to Nelder and Mead [16]. For the reflection coefficient, the expansion coefficient and the contraction coefficient of the algorithm we have chosen the values 1.0, 2.0 and 0.5 , respectively.
} 
Table 2 Parameters of the local long run risk premium functions

\begin{tabular}{lllll}
\hline & $d_{x}$ & $d_{y}$ & $l_{x}$ & $l_{y}$ \\
\hline Constant function & -0.0112 & 0.0779 & & \\
Step function & -0.0112 & 0.0779 & -0.0081 & -0.0088 \\
Linear function & -0.0151 & 0.1672 & -0.0081 & -0.0088 \\
\hline
\end{tabular}

we have been inspired by the framework developed by the Frauenhofer ITWM on behalf of PIA to classify PRIIPs into chance-risk classes (see Korn and Wagner [15]). Their model represents the industry standard for PRIIP calculations. The latest OECD forecasts regarding the 31.12.2019 for the longest horizon, which is the fourth quarter of 2021 , amount to $-0.4 \%$ and $0.4 \%$, respectively. ${ }^{2}$ For the long term interest rate forecasts, which are needed to calibrate the step and the linear function, we take the average of monthly 3-month and 10-year interest rates over the last 15 years also published by the OECD. This is a valid approach if interest rates follow a stationary process, because in this case historical data can be considered as a random sample from the corresponding interest rate distribution. Hull et al. [13] point out that this approach is questionable if monetary and fiscal policies are expected to be materially different from those in the past. Nevertheless any other model based on historical data would be questionable and the user of the model can alternatively provide personal estimates or an expert judgment. The historical average amounts to $1.08 \%$ for the 3 -month and $1.84 \%$ for the 10 -year interest rate and as we assume these forecasts to be a long run average we set the forecasting horizon to 40 years- the modelling horizon. We further set $\tau$ to 24 months, which is the forecasting horizon of the short term OECD forecasts.

Table 2 shows the calibration results for the three local long run risk premium function types.

The values of $d_{x}$ and $d_{y}$ coincide for the constant and the step function as the same interest rate forecasts have been used in the calibration process. But in contrast to the step function, which takes the values of $l_{x}$ and $l_{y}$ after 24 months, the constant function stays constant for the whole modelling horizon. It also appears that the step and the linear function take the same values for $l_{x}$ and $l_{y}$. But there is a slight difference as their functional forms differ in the first two years, which influences the absolute risk premium in future time points. This influence decreases in time, such that the difference is negligible as we calibrated $l_{x}$ and $l_{y}$ to forecasts with an forecasting horizon of 40 years.

Figures 2, 3 and 4 visualize for the three calibrated variants of the Gauss $2++$ model the development of the expectation of the short-rate, the 10-year and the 20 -year interest rate for forecasting horizons of up to 40 years. The solid line represents the expectation under the risk neutral measure, the dashed line shows the expected values under the real world measure.

\footnotetext{
2 https://stats.oecd.org: The rounded numbers can be found, if one selects the data for the Economic Outlook N.106 of November 2019 in the section Economic Projections.
} 


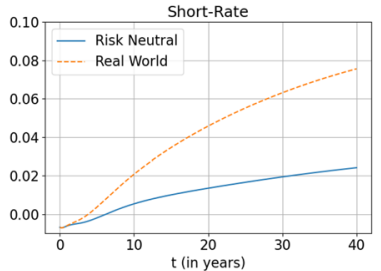

(a)

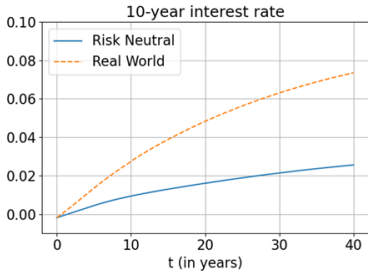

(b)

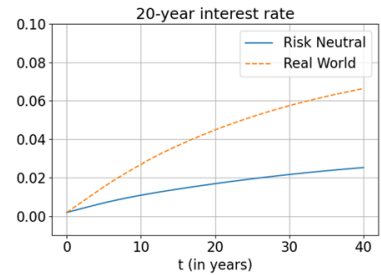

(c)

Fig. 2 Constant function: expected values of the short-rate, the 10 -year and the 20 -year interest rate under the risk neutral and the real world measure

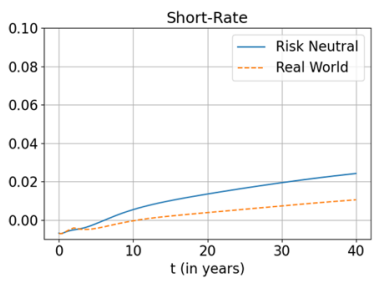

(a)

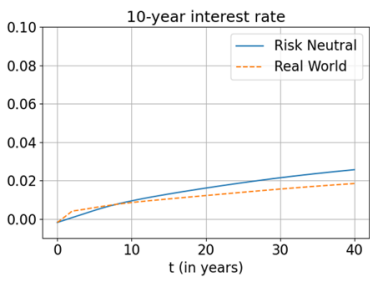

(b)

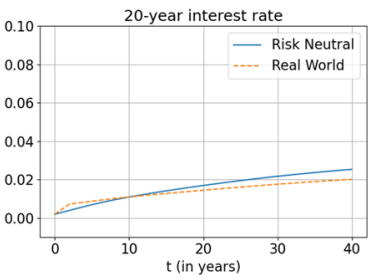

(c)

Fig. 3 Step function: expected values of the short-rate, the 10-year and the 20-year interest rate under the risk neutral and the real world measure

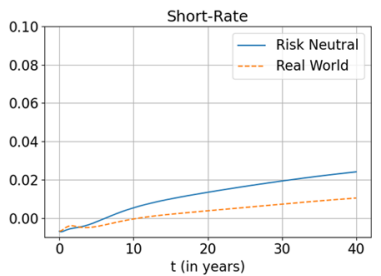

(a)

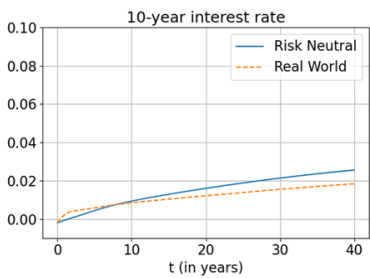

(b)

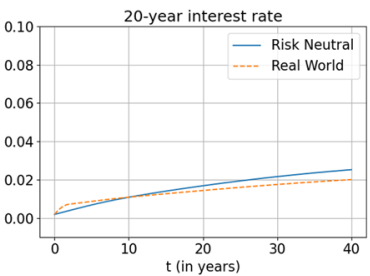

(c)

Fig. 4 Linear function: expected values of the short-rate, the 10-year and the 20-year interest rate under the risk neutral and the real world measure

For the variant of the Gauss $2++$ model, which uses the constant function as the local long run risk premium function, the expected real world interest rates lie above the risk neutral expectation. This means, that a risk seeking behaviour of the investors is assumed for the whole modelling period, because an investor accepts a lower expected return for a corresponding bond if the interest rates are expected to be higher in the real world compared to the risk neutral world. Ahmad and Wilmott [1] show that there have been time periods where investors seem to have historically behaved in this way. But in general investors are assumed to be risk averse and therefore interest rates should be lower in the real world than in the risk neutral world, which is an opposite behaviour to equity prices (see, e.g. Hull et al. [13]). For the other two variants of the Gauss $2++$ model the expected 
Fig. 5 Absolute risk premium function for the variants of the Gauss $2++$ model

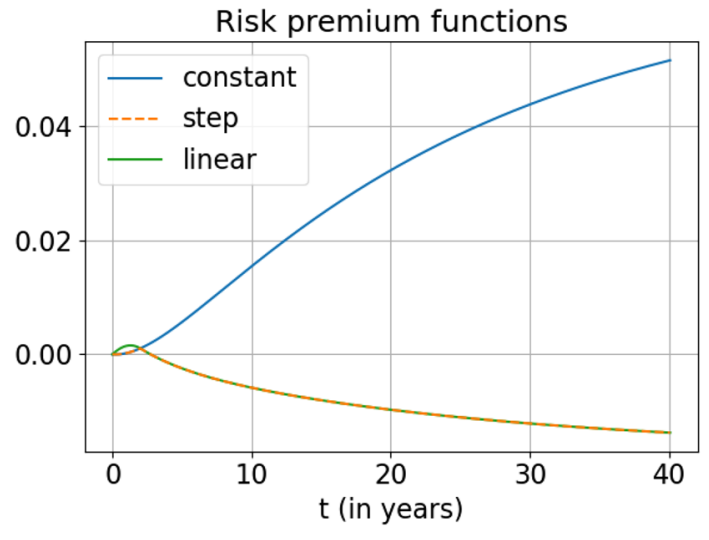

real world interest rates lie also above the risk neutral interest rates in the short horizon but below in the long horizon. This assumption of risk seeking behaviour in the short horizon stems from the quite high forecasts of the OECD for the short horizon, but it might be valid in the current market situation. In contrast to the constant case, which keeps this risk seeking behaviour assumption for the whole modelling horizon, in the long run the other two variants of the Gauss $2++$ model assume in this calibration a risk averse behaviour. The difference between the step and the linear function is only visible in the short horizon. While the step function has a kink in the expectation after $\tau$ years, the linear function is smoother due to its condition that the derivative of the absolute risk premium function exists at this time point.

Furthermore, the absolute difference in the risk neutral and real world expectations decreases for interest rates with longer maturities. This results from the less variation of interest rates with longer maturities, which is an implicit model characteristic of the Gauss $2++$ model and is supported by historical data as well. A risk premium is therefore higher (less negative) for a risk averse and lower (less positive) for a risk seeking investor in an arbitrage free market.

Figure 5 shows the absolute risk premium functions of the short-rate for all three modelling types.

It can be observed that for the constant and the step function the absolute risk premium is the same up to year 2. After that year the Gauss $2++$ variant with the step function has a kink in the absolute risk premium as the local long run risk premium changes to a different level, while the modelling case with the constant function continuous to approach the long term risk premium determined by the short term interest rate forecasts. The modelling case with the linear function results in a different risk premium for the first 2 years, but approaches-without a kink- the same long term risk premium as the step function.

All three functions intersect after 2 years as this is the forecasting horizon of the short term interest rate forecasts, which were used for the calibration. The absolute risk premium at this time point must be the same for all modelling cases such that the expected interested rates of the model coincide with the forecasts. 
Fig. 6 The share of inverse yield curves for the Gauss $2++$ model under the risk neutral measure and under the real world measure using a constant, a step and a linear function for the market price of risk

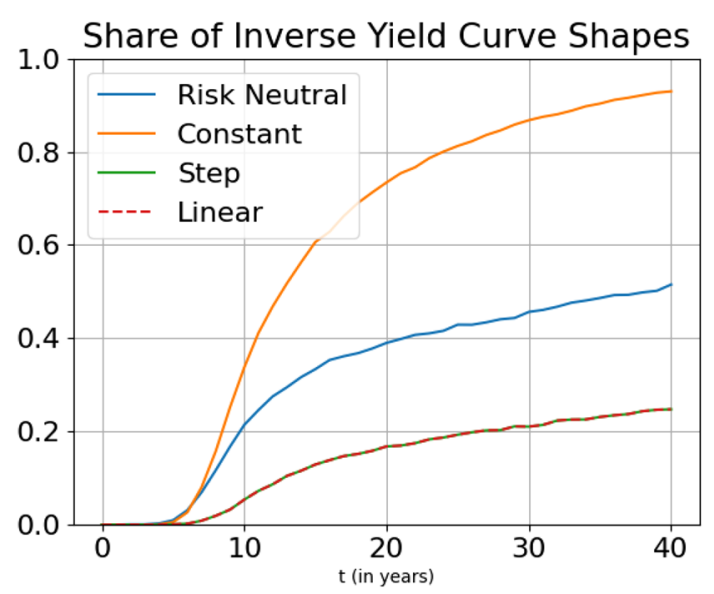

We further investigated the resulting yield curve shapes of the three variants of the Gauss $2++$ model. The variant, which uses a constant function, represents the industry standard regarding PRIIP calculations (see Korn and Wagner [15]). An unpleasent feature of this model is the unrealistic high frequency of inverse yield curves with growing time (see Diez and Korn [7]). In their paper they show that for the 2-Factor Vasicek model the number of inverse yield curves can be reduced by assuming a negative risk premium. The share of inverse yield curves in our calibration of the three variants were investigated in a simulation study. We simulated 10,000 yield curve paths with each calibrated model and counted the number of yield curves, which have a higher 1-year interest rate than a 30-year interest rate. The result is visualized in Fig. 6. We can see a similar behaviour as described in the paper of Diez and Korn [7]. The variant with the constant function, which has a positive risk premium over the modelling horizon, shows an unrealistic high share of inverse yield curves. The other two variants have a negative risk premium and decrease the number of inverse yield curves in the long run compared to the risk neutral case. Using the step or the linear function for the risk premium results therefore not only in more realistic interest rates but also in more realistic yield curve shapes in the long horizon.

\subsection{Backtest}

In this subsection the different variants of the Gauss $2++$ model calibrated on a quarterly basis over the last 3 years are compared.

As in Sect. 4.1 interest rate swaps and swaption volatilities have been used for the risk neutral calibration of the Gauss $2++$ model. To calibrate the parameters of the local long run risk premium functions in the second calibration step short term interest rate forecasts published by the OECD and a long term average have been used. The forecasts are shown in Table 3. The calibration results of the parameters of the Gauss $2++$ model under the risk neutral measure and of the local long run risk 
Table 3 Interest rate forecasts of the OECD and historical average of the 3-month and the 10-year interest rate

\begin{tabular}{|c|c|c|c|c|c|}
\hline \multirow[t]{2}{*}{ Date } & \multicolumn{3}{|c|}{ Short term interest rate forecasts } & \multicolumn{2}{|c|}{ Historical average } \\
\hline & $\begin{array}{l}\text { Forecasting horizon } \\
\text { (in months) }\end{array}$ & $\begin{array}{l}\text { 3-m IR } \\
(\text { in } \%)\end{array}$ & $\begin{array}{l}10-y \text { IR } \\
(\text { in } \%)\end{array}$ & $\begin{array}{l}\text { 3-m IR } \\
(\text { in } \%)\end{array}$ & $\begin{array}{l}10-y \text { IR } \\
(\text { in } \%)\end{array}$ \\
\hline 30.09 .2019 & 15 & -0.3 & 1.0 & 1.13 & 1.91 \\
\hline 30.06 .2019 & 18 & -0.3 & 1.0 & 1.18 & 1.98 \\
\hline 31.03.2019 & 21 & -0.2 & 1.6 & 1.22 & 2.04 \\
\hline 31.12 .2018 & 24 & -0.2 & 1.6 & 1.26 & 2.10 \\
\hline 30.09 .2018 & 15 & -0.2 & 1.3 & 1.31 & 2.16 \\
\hline 30.06 .2018 & 18 & -0.2 & 1.3 & 1.35 & 2.23 \\
\hline 31.03.2018 & 21 & -0.3 & 1.4 & 1.39 & 2.30 \\
\hline 31.12 .2017 & 24 & -0.3 & 1.4 & 1.44 & 2.36 \\
\hline 30.09 .2017 & 15 & -0.3 & 1.6 & 1.48 & 2.43 \\
\hline 30.06 .2017 & 18 & -0.3 & 1.6 & 1.52 & 2.50 \\
\hline 31.03 .2017 & 21 & -0.3 & 1.6 & 1.57 & 2.56 \\
\hline 31.12 .2016 & 24 & -0.3 & 1.6 & 1.63 & 2.63 \\
\hline
\end{tabular}

https://stats.oecd.org: The rounded numbers can be found, if one selects the annual interest rate forecasts of the corresponding Economic Outlook in the section Economic Projections

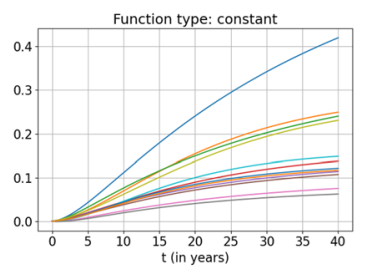

(a)

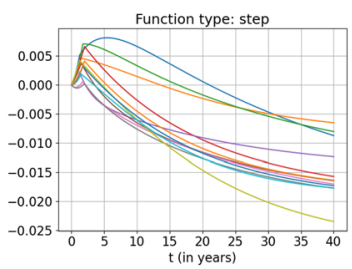

(b)

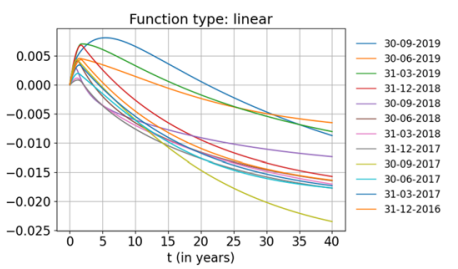

(c)

Fig. 7 Absolute risk premium functions

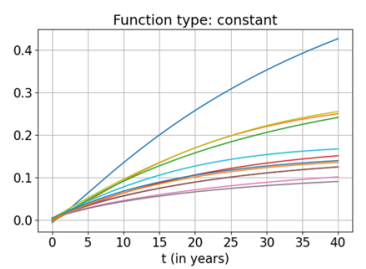

(a)

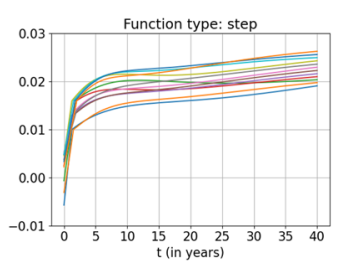

(b)

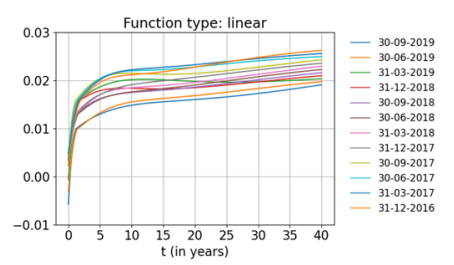

(c)

Fig. 8 Development of the expectation of the 10-year interest rate over the modelling horizon for all three variants of the Gauss $2++$ model 
premium function for each variant of the Gauss $2++$ model can be found in Tables 4, 5, 6 and 7 in "Appendix 6".

For each calibration the absolut risk premium function of the short-rate and the development of the expected 10-year interest rate have been calculated and visualised in Figs. 7 and 8.

The absolute risk premium function of the short-rate for the Gauss $2++$ model, which uses the constant function for the local long run risk premium, depends highly on the risk neutral calibration results and the forecasts of the OECD. An unfavorable combination of market data and interest rate forecasts can lead to a high value for the local long run risk premium. This value might be reasonable to meet the short term forecasts used for the calibration, but as it stays constant over time it is the value the absolute risk premium is converging to. Therefore, this problem can strike through if the modelling horizon is much longer than the forecasting horizon of the interest rates used for the calibration. In this case a timevarying local long run risk premium function, which can be calibrated to a short and a long term forecast, is more convenient to regularize the risk premium. As it can be seen in Fig. 7 the variants of the Gauss $2++$ model, which use the step or the linear function for the local long run risk premium, produce more stable risk premiums in the long horizon. In each calibration the absolute risk premium is positive in the first years, which presumes a risk seeking behaviour of the investors, but in the long horizon the absolute risk premium lies between -0.5 and $-2.5 \%$ representing a risk averse market. Also the interest rate distribution in the long horizon is more stable. Figure $8 b$, c show that the expectation of the 10-year interest rate in the long horizon change only little in each calibration according to the historical average, which was used for the long term interest rate forecast.

\section{Conclusion}

As the Gauss $2++$ model is often used for pricing purposes, the focus in the literature lies on the evolution of interest rates under the risk neutral measure $\mathbb{Q}$. But regarding risk management and forecasting applications the model under the real world measure is needed. In this paper we introduced a framework to apply the model under both measures in a consistent manner. This framework first conducts a calibration under the risk neutral measure and then determines the change of measure such that it is possible to switch between the risk neutral and the real world. We showed that according to Girsanov this change of measure can be specified by any time-dependent function without loosing the analytic tractability of, e.g. zero-coupon bond prices. Hull et al. [13] argue that because of unobserved risk factors, which are not included in the model, a time-varying function should be used, because otherwise unrealistic interest rates in the long forecasting horizon could be reached. We therefore compared the industry standard, which uses a constant function to model the change of measure, with two variants, which use either a step or a linear function. These functions are the simplest extensions of the constant function to a time-varying function without increasing the computational effort much. By accounting for different risk premiums in the short and in the long horizon the 
time-varying functions result in much more stable interest rate forecasts in the long run if calibrated at different valuation dates. From a macroeconomical point of view it makes sense that current market fluctuations should not influence interest rate forecasts in the long horizon, e.g. in 40 years, much. This would also imply that risk measures calculated with the Gauss $2++$ model, which uses one of the time-varying functions for the change of measure, would be more consistent if estimated at different valuation time points.

We further investigated the yield curve shapes by conducting a simulation study. The result is in line with the findings of Diez and Korn [7] for the 2-Factor Vasicek model. Assuming a positive risk premium - as it was the case in our calibration for the constant function - the number of inverse yield curves increases compared to the risk neutral case. This also replicates the problem of too many inverse yield curves in the insurance industry for PRIIP calculations (see Diez and Korn [7]). The other two variants represented in this paper, which apply a time-varying function for the market price of risk, assume a negative risk premium in the long run and have a much lower amount of inverse yield curves. Using a step or a linear function for the market price of risk, therefore, not only leads to more realistic interest rates in the long run, but also creates more realistic yield curve shapes.

\section{Appendix 1: Bond price dynamic under the risk neutral measure}

By defining

$$
A(t, T)=-\int_{t}^{T} \varphi(s) d s+\frac{1}{2} V(t, T),
$$

the price of a zero-coupon bond $P(t, T)$ at time point $t$ and maturity $T$ can be calculated for the Gauss $2++$ model under the risk neutral measure $\mathbb{Q}$ by

$$
P(t, T)=e^{A(t, T)-B(a, t, T) x(t)-B(b, t, T) y(t)} .
$$

A proof of this formula can be found in Brigo and Mercurio [2]. The derivatives of $A(t, T)$ and $V(t, T)$ with respect to the first entry and of $B(z, t, T)$ with respect to the second entry are given by

$$
\begin{aligned}
A^{\prime}(t, T) & =\varphi(t)+\frac{1}{2} V^{\prime}(t, T), \\
V^{\prime}(t, T) & =-\sigma^{2} B(a, t, T)^{2}-\eta^{2} B(b, t, T)^{2}-2 \sigma \eta \rho B(a, t, T) B(b, t, T), \\
B^{\prime}(z, t, T) & =-e^{-z(T-t)} .
\end{aligned}
$$

Furthermore, it holds

$$
B(z, t, T) z-B^{\prime}(z, t, T)=1 .
$$

To calculate the zero-coupon bond price dynamic, we apply Ito's formula to (12), i.e. 


$$
\begin{aligned}
d P(t, T)= & P(t, T)[A(t, T)-B(a, t, T) x(t)-B(b, t, T) y(t)]^{\prime} d t+P(t, T)(-B(a, t, T)) d x(t) \\
& +P(t, T)(-B(b, t, T)) d y(t) \\
& +\frac{1}{2} P(t, T) B(a, t, T)^{2} \sigma^{2} d t \\
& +\frac{1}{2} P(t, T) B(b, t, T)^{2} \eta^{2} d t \\
& +P(t, T) B(a, t, T) B(b, t, T) \sigma \eta \rho d t \\
= & P(t, T)\left[A^{\prime}(t, T)-B^{\prime}(a, t, T) x(t)-B^{\prime}(b, t, T) y(t)+B(a, t, T) a x(t)+B(b, t, T) b y(t)\right. \\
& +\frac{1}{2} B(a, t, T)^{2} \sigma^{2}+\frac{1}{2} B(b, t, T)^{2} \eta^{2} \\
& +B(a, t, T) B(b, t, T) \sigma \eta \rho] d t \\
& -B(a, t, T) P(t, T) \sigma d W^{1}(t) \\
& -B(b, t, T) P(t, T) \eta d W^{2}(t) \\
= & P(t, T)[\varphi(t)+x(t)+y(t)] d t-B(a, t, T) P(t, T) \sigma d W^{1}(t)-B(b, t, T) P(t, T) \eta d W^{2}(t) \\
= & P(t, T) r(t) d t-B(a, t, T) P(t, T) \sigma d W^{1}(t)-B(b, t, T) P(t, T) \eta d W^{2}(t) .
\end{aligned}
$$

\section{Appendix 2: The dynamics of the Gauss2++ factors $x$ and $y$ under the real world measure}

The dynamics of the two processes $x$ and $y$ under the risk neutral measure $\mathbb{Q}$ can be expressed in terms of two independent Brownian motions $\widehat{W}^{1}$ and $\widehat{W}^{2}$, i.e.

$$
\begin{aligned}
& d x(t)=-a x(t) d t+\sigma d \widehat{W}^{1}(t), \\
& d y(t)=-b y(t) d t+\eta \rho d \widehat{W}^{1}(t)+\eta \sqrt{\left(1-\rho^{2}\right)} d \widehat{W}^{2}(t),
\end{aligned}
$$

where

$$
\begin{aligned}
& d W^{1}(t)=d \widehat{W}^{1}(t), \\
& d W^{2}(t)=\rho d \widehat{W}^{1}(t)+\sqrt{\left(1-\rho^{2}\right)} d \widehat{W}^{2}(t) .
\end{aligned}
$$

According to Girsanov's theorem, as $\widehat{\boldsymbol{W}}=\left(\widehat{W}^{1}, \widehat{W}^{2}\right)$ is a standard 2-dimensional Brownian motion and let $(\boldsymbol{\Phi}(t))_{t \in[0, \mathcal{T}] \breve{W}}=\left(\Phi^{1}(t), \Phi^{2}(t)\right)_{t \in[0, \mathcal{T}]}$ be a progressive and square-integrable process, the process $\breve{W}$ defined by

$$
\breve{\boldsymbol{W}}(t):=\widehat{\boldsymbol{W}}(t)+\int_{0}^{t} \boldsymbol{\Phi}(s) d s
$$

is a standard 2-dimensional Brownian motion under a new measure, which we call $\mathbb{P}$ and declare to be the real world measure. This means that the dynamic of the two Brownian motion $\widehat{W}^{1}$ and $\widehat{W}^{2}$ under the real world measure $\mathbb{P}$ is given by 


$$
\begin{aligned}
& d \widehat{W}^{1}(t)=d \breve{W}^{1}(t)-\Phi^{1}(t) d t \\
& d \widehat{W}^{2}(t)=d \breve{W}^{2}(t)-\Phi^{2}(t) d t .
\end{aligned}
$$

Therefore, the dynamics of the two processes $x$ and $y$ under the real world measure are then given by

$$
\begin{aligned}
d x(t)= & {\left[-\Phi^{1}(t) \sigma-a x(t)\right] d t+\sigma d \breve{W}^{1}(t), } \\
d y(t)= & {\left[-\Phi^{1}(t) \eta \rho-\Phi^{2}(t) \eta \sqrt{\left(1-\rho^{2}\right)}-b y(t)\right] d t+\eta \rho d \breve{W}^{1}(t) } \\
& +\eta \sqrt{\left(1-\rho^{2}\right)} d \breve{W}^{2}(t) .
\end{aligned}
$$

If we specify $\Phi(t)$ as in (3) this simplifies to

$$
\begin{aligned}
& d x(t)=a\left(d_{x}(t)-x(t)\right) d t+\sigma d \breve{W}^{1}(t), \\
& d y(t)=b\left(d_{y}(t)-y(t)\right) d t+\eta \rho d \breve{W}^{1}(t)+\eta \sqrt{\left(1-\rho^{2}\right)} d \breve{W}^{2}(t) .
\end{aligned}
$$

Representing the dynamics by two correlated Brownian motions $\widetilde{W}^{1}$ and $\widetilde{W}^{2}$ results in the equations given in (4) and (5).

\section{Appendix 3: Bond price dynamic under the real world measure}

The dynamic of a zero-coupon bond price $P(t, T)$ under the risk neutral measure $\mathbb{Q}$ expressed by the two independent Brownian motions $\widehat{W}^{1}$ and $\widehat{W}^{2}$ is given by

$$
\begin{aligned}
d P(t, T)= & P(t, T) r(t) d t-P(t, T) B_{\tau}(a) \sigma d \widehat{W}^{1}(t)-P(t, T) B_{\tau}(b) \eta \rho d \widehat{W}^{1}(t) \\
& -P(t, T) B_{\tau}(b) \eta \sqrt{\left(1-\rho^{2}\right)} d \widehat{W}^{2}(t), \\
= & P(t, T) r(t) d t-\left[P(t, T) B_{\tau}(a) \sigma+P(t, T) B_{\tau}(b) \eta \rho\right] d \widehat{W}^{1}(t) \\
& -P(t, T) B_{\tau}(b) \eta \sqrt{\left(1-\rho^{2}\right)} d \widehat{W}^{2}(t) .
\end{aligned}
$$

Applying Girsanov's theorem as in "Appendix 2" the dynamic under the real world measure $\mathbb{P}$ amounts to 


$$
\begin{aligned}
d P(t, T)= & P(t, T) r(t) d t-\left[P(t, T) B_{\tau}(a) \sigma+P(t, T) B_{\tau}(b) \eta \rho\right] d \widehat{W}^{1}(t) \\
& -P(t, T) B_{\tau}(b) \eta \sqrt{\left(1-\rho^{2}\right)} d \widehat{W}^{2}(t) \\
= & P(t, T)\left[r(t)+\left(B_{\tau}(a) \sigma+B_{\tau}(b) \eta \rho\right)\left(-\frac{a d_{x}(t)}{\sigma}\right)\right. \\
& \left.+B_{\tau}(b) \eta \sqrt{\left(1-\rho^{2}\right)}\left(-\frac{b d_{y}(t)}{\eta \sqrt{\left(1-\rho^{2}\right)}}+\frac{\rho a d_{x}(t)}{\sigma \sqrt{\left(1-\rho^{2}\right)}}\right)\right] d t \\
& -\left[P(t, T) B_{\tau}(a) \sigma+P(t, T) B_{\tau}(b) \eta \rho\right] d \breve{W}^{1}(t) \\
& -P(t, T) B_{\tau}(b) \eta \sqrt{\left(1-\rho^{2}\right)} d \breve{W}^{2}(t) \\
= & P(t, T)\left[r(t)-B_{\tau}(a) a d_{x}(t)-B_{\tau}(b) b d_{y}(t)\right] d t \\
& -\left[P(t, T) B_{\tau}(a) \sigma+P(t, T) B_{\tau}(b) \eta \rho\right] d \breve{W}^{1}(t) \\
& -P(t, T) B_{\tau}(b) \eta \sqrt{\left(1-\rho^{2}\right)} d \breve{W}^{2}(t) .
\end{aligned}
$$

Representing the dynamic by two correlated Brownian motions $\widetilde{W}^{1}$ and $\widetilde{W}^{2}$ results in the equation given in (8).

\section{Appendix 4: Individual discount rate for the zero-coupon bonds in the real world}

Proof To proof that $\frac{P(t, T)}{X(t, T)}$ is indeed a martingale we calculate the dynamic of the discounted price process. 


$$
\begin{aligned}
d \frac{P(t, T)}{X(t)}= & d\left(\frac{1}{X(t)} \cdot P(t, T)\right) \\
= & \frac{1}{X(t)} d P(t, T)+P(t, T) d \frac{1}{X(t)}+d\left\langle P(t, T), \frac{1}{X(t)}\right\rangle \\
= & \frac{1}{X(t)} d P(t, T)-\frac{P(t, T)}{X(t)}\left[r(t)-B(a, t, T) a d_{x}(t)-B(b, t, T) b d_{y}(t)\right] d t \\
= & \frac{P(t, T)}{X(t)}\left[r(t)-B(a, t, T) a d_{x}(t)-B(b, t, T) b d_{y}(t)\right] d t \\
& -\frac{P(t, T)}{X(t)} B(a, t, T) \sigma d \widetilde{W}^{1}(t)-\frac{P(t, T)}{X(t)} B(b, t, T) \eta d \widetilde{W}^{2}(t) \\
& -\frac{P(t, T)}{X(t)}\left[r(t)-B(a, t, T) a d_{x}(t)-B(b, t, T) b d_{y}(t)\right] d t \\
= & -\frac{P(t, T)}{X(t)} B(a, t, T) \sigma d \widetilde{W}^{1}(t)-\frac{P(t, T)}{X(t)} B(b, t, T) \eta d \widetilde{W}^{2}(t)
\end{aligned}
$$

\section{Appendix 5: Bond price formula under the real world measure}

To calculate the price of a zero-coupon bond under the real world measure $\mathbb{P}$, the distribution of

$$
\exp \left(-\int_{t}^{T}\left(r(u)-B(a, u, T) a d_{x}(u)-B(b, u, T) b d_{y}(u)\right) d u\right)
$$

has to be determined. In the following we show, that the integral in the exponent is normaly distributed and calculate the mean and the variance of

$$
I(t, T):=\int_{t}^{T}\left(r(u)-B(a, u, T) a d_{x}(u)-B(b, u, T) b d_{y}(u)\right) d u .
$$

We first concentrate on the integral over the short-rate $r(s)$, which is a sum of the $x$ and the $y$-process and a deterministic function

$$
r(s)=x(s)+y(s)+\varphi(s) .
$$

The integral over the process $x$ is given by 


$$
\begin{aligned}
\int_{t}^{T} x(u) d u= & \int_{t}^{T}\left(x(t) e^{-a(u-t)}+\int_{t}^{u} a e^{-a(u-s)} d_{x}(s) d s\right. \\
& \left.+\int_{t}^{u} \sigma e^{-a(u-s)} d \tilde{W}^{1}(s)\right) d u \\
= & \underbrace{\int_{t}^{T} x(t) e^{-a(u-t)} d u}_{(1)}+\underbrace{\int_{t}^{T} \int_{t}^{u} a e^{-a(u-s)} d_{x}(s) d s d u}_{(2)} \\
& +\underbrace{}_{\int_{t}^{T} \int_{t}^{u} \sigma e^{-a(u-s)} d \tilde{W}^{1}(s) d u}
\end{aligned}
$$

(3)

The first integral amounts to

$$
\text { (1) }=x(t) \int_{t}^{T} e^{-a(u-t)} d u=x(t)\left[-\frac{1}{a} e^{-a(u-t)}\right]_{t}^{T}=x(t) \frac{1-e^{-a(T-t)}}{a} \text {. }
$$

For the second integral we use the integration by parts formula

$$
\begin{aligned}
(2) & =\int_{t}^{T}\left(\int_{t}^{u} e^{a s} d_{x}(s) d s\right) a e^{-a u} d u \\
& =a \int_{t}^{T}\left(\int_{t}^{u} e^{a s} d_{x}(s) d s\right) d_{u}\left(\int_{t}^{u} e^{-a v} d v\right) \\
& =a\left[\left(\int_{t}^{T} e^{a u} d_{x}(u) d u\right)\left(\int_{t}^{T} e^{-a v} d v\right)-\int_{t}^{T}\left(\int_{t}^{u} e^{-a v} d v\right) e^{a u} d_{x}(u) d u\right] \\
& =a\left[\int_{t}^{T}\left(\int_{u}^{T} e^{-a v} d v\right) e^{a u} d_{x}(u) d u\right] \\
& =\int_{t}^{T}\left(1-e^{-a(T-u)}\right) d_{x}(u) d u \\
& =\int_{t}^{T} a B(a, u, T) d_{x}(u) d u .
\end{aligned}
$$

For the third integral we again use the integration by parts formula 


$$
\begin{aligned}
3 & =\sigma \int_{t}^{T}\left(\int_{t}^{u} e^{a s} d \widetilde{W}^{1}(s)\right) a e^{-a u} d u \\
& =\sigma \int_{t}^{T}\left(\int_{t}^{u} e^{a s} d \widetilde{W}^{1}(s)\right) d_{u}\left(\int_{t}^{u} e^{-a v} d v\right) \\
& =\sigma\left[\left(\int_{t}^{T} e^{a u} d \widetilde{W}^{1}(u)\right)\left(\int_{t}^{T} e^{-a v} d v\right)-\int_{t}^{T}\left(\int_{t}^{u} e^{-a v} d v\right) e^{a u} d \widetilde{W}^{1}(u)\right] \\
& =\sigma\left[\int_{t}^{T}\left(\int_{u}^{T} e^{-a v} d v\right) e^{a u} d \widetilde{W}^{1}(u)\right] \\
& =\sigma \int_{t}^{T}\left[-\frac{e^{-a v}}{a}\right]_{u}^{T} e^{a u} d \widetilde{W}^{1}(u) \\
& =\frac{\sigma}{a} \int_{t}^{T}\left(1-e^{-a(T-u)}\right) d \widetilde{W}^{1}(u)
\end{aligned}
$$

The corresponding expressions for $\int_{t}^{T} y(u) d u$ can be obtained analogously. We observe that the results of integral (2) for $\int_{t}^{T} x(u) d u$ and $\int_{t}^{T} y(u) d u$ cancel out with the last two terms in equation (13). Therefore it remains

$$
\begin{aligned}
I(t, T)= & \int_{t}^{T} \varphi(u) d u+\frac{1-e^{-a(T-t)}}{a} x(t)+\frac{1-e^{-b(T-t)}}{b} y(t) \\
& +\frac{\sigma}{a} \int_{t}^{T}\left(1-e^{-a(T-u)}\right) d \widetilde{W}^{1}(u)+\frac{\eta}{b} \int_{t}^{T}\left(1-e^{-b(T-u)}\right) d \widetilde{W}^{2}(u) .
\end{aligned}
$$

As $\widetilde{\boldsymbol{W}}=\left(\widetilde{W}^{1}, \widetilde{W}^{2}\right)$ is a 2-dimensional Brownian motion under $\mathbb{P}, I(t, T)$ is normally distributed and the mean and the variance can be easily retrieved resulting in (9) and (10).

\section{Appendix 6: Tables of backtest results}

See Tables 4, 5, 6 and 7. 
Table 4 Calibration results of the risk neutral calibration on a quarterly basis from 31.12.2016 to 30.09 .2019

Table 5 Quarterly calibration results for the constant local long run risk premium functions from 31.12.2016 to 30.09.2019

\begin{tabular}{llllll}
\hline Date & $a$ & $b$ & $\sigma$ & $\eta$ & $\rho$ \\
\hline 30.09 .2019 & 0.2694 & 0.0269 & 0.0121 & 0.0089 & -0.8950 \\
30.06 .2019 & 0.1216 & 0.0628 & 0.0363 & 0.0283 & -0.9687 \\
31.03 .2019 & 0.3978 & 0.0331 & 0.0333 & 0.0091 & -0.8576 \\
31.12 .2018 & 0.1628 & 0.0521 & 0.0183 & 0.0154 & -0.8629 \\
30.09 .2018 & 0.6100 & 0.0429 & 0.0459 & 0.0104 & -0.8722 \\
30.06 .2018 & 0.2901 & 0.0459 & 0.0104 & 0.0112 & -0.9941 \\
31.03 .2018 & 0.5120 & 0.0386 & 0.0142 & 0.0097 & -1.0000 \\
31.12 .2017 & 0.3803 & 0.0471 & 0.0236 & 0.0120 & -0.8854 \\
30.09 .2017 & 0.0880 & 0.0655 & 0.0421 & 0.0460 & -0.9938 \\
30.06 .2017 & 0.1260 & 0.0890 & 0.0504 & 0.0517 & -0.9963 \\
31.03 .2017 & 0.2940 & 0.0581 & 0.0152 & 0.0146 & -0.9984 \\
31.12 .2016 & 0.2427 & 0.0606 & 0.0178 & 0.0173 & -1.0000 \\
\hline
\end{tabular}

\begin{tabular}{lll}
\hline Date & $d_{x}$ & $d_{y}$ \\
\hline 30.09 .2019 & -0.0676 & 0.7400 \\
30.06 .2019 & -0.2848 & 0.5787 \\
31.03 .2019 & -0.0267 & 0.3636 \\
31.12 .2018 & -0.0539 & 0.2182 \\
30.09 .2018 & -0.0107 & 0.1518 \\
30.06 .2018 & -0.0173 & 0.1481 \\
31.03 .2018 & -0.0112 & 0.1099 \\
31.12 .2017 & -0.0150 & 0.0913 \\
30.09 .2017 & -0.7023 & 0.9836 \\
30.06 .2017 & -0.3883 & 0.5497 \\
31.03 .2017 & -0.0330 & 0.1710 \\
31.12 .2016 & -0.0405 & 0.1725 \\
\hline
\end{tabular}


Table 6 Quarterly calibration results for the step local long run risk premium functions from 31.12.2016 to 30.09 .2019

Table 7 Quarterly calibration results for the linear local long run risk premium functions from 31.12.2016 to 30.09.2019

\begin{tabular}{lllll}
\hline Date & $d_{x}$ & $d_{y}$ & $l_{x}$ & $l_{y}$ \\
\hline 30.09 .2019 & -0.0676 & 0.7400 & -0.0090 & -0.0129 \\
30.06 .2019 & -0.2848 & 0.5787 & -0.0376 & -0.0292 \\
31.03 .2019 & -0.0267 & 0.3636 & -0.0114 & -0.0034 \\
31.12 .2018 & -0.0539 & 0.2182 & -0.0163 & -0.0029 \\
30.09 .2018 & -0.0107 & 0.1518 & -0.0101 & -0.0047 \\
30.06 .2018 & -0.0173 & 0.1481 & -0.0107 & -0.0090 \\
31.03 .2018 & -0.0112 & 0.1099 & -0.0087 & -0.0129 \\
31.12 .2017 & -0.0150 & 0.0913 & -0.0099 & -0.0111 \\
30.09 .2017 & -0.7023 & 0.9836 & -0.0364 & -0.0087 \\
30.06 .2017 & -0.3883 & 0.5497 & -0.0423 & -0.0233 \\
31.03 .2017 & -0.0330 & 0.1710 & -0.0131 & -0.0068 \\
31.12 .2016 & -0.0405 & 0.1725 & -0.0154 & -0.0033 \\
\hline
\end{tabular}

\begin{tabular}{lllll}
\hline Date & $d_{x}$ & $d_{y}$ & $l_{x}$ & $l_{y}$ \\
\hline 30.09 .0219 & -0.1332 & 1.5015 & -0.0090 & -0.0129 \\
30.06 .2019 & -0.5474 & 1.1457 & -0.0376 & -0.0292 \\
31.03 .2019 & -0.0461 & 0.7377 & -0.0114 & -0.0034 \\
31.12 .2018 & -0.0959 & 0.4471 & -0.0163 & -0.0029 \\
30.09 .2018 & -0.0114 & 0.3111 & -0.0101 & -0.0047 \\
30.06 .2018 & -0.0250 & 0.3087 & -0.0107 & -0.0090 \\
31.03 .2018 & -0.0144 & 0.2355 & -0.0087 & -0.0129 \\
31.12 .2017 & -0.0216 & 0.1970 & -0.0099 & -0.0111 \\
30.09 .2017 & -1.3930 & 1.9854 & -0.0364 & -0.0087 \\
30.06 .2017 & -0.7567 & 1.1001 & -0.0423 & -0.0233 \\
31.03 .2017 & -0.0567 & 0.3550 & -0.0131 & -0.0068 \\
31.12 .2016 & -0.0700 & 0.3556 & -0.0154 & -0.0033 \\
\hline & & & &
\end{tabular}

Acknowledgements This research was supported by ROKOCO predictive analytics GmbH. We also thank the two anonymous reviewers for helpful comments and suggestions.

Funding Open Access funding enabled and organized by Projekt DEAL.

Open Access This article is licensed under a Creative Commons Attribution 4.0 International License, which permits use, sharing, adaptation, distribution and reproduction in any medium or format, as long as you give appropriate credit to the original author(s) and the source, provide a link to the Creative Commons licence, and indicate if changes were made. The images or other third party material in this article are included in the article's Creative Commons licence, unless indicated otherwise in a credit line to the material. If material is not included in the article's Creative Commons licence and your intended use is not permitted by statutory regulation or exceeds the permitted use, you will need to obtain permission directly from the copyright holder. To view a copy of this licence, visit http://creativecommons.org/licen ses/by/4.0/. 


\section{References}

1. Ahmad R, Wilmott P (2006) The market price of interest-rate risk: measuring and modelling fear and greed in the fixed-income markets. Wilmott Mag 30:64-70

2. Brigo D, Mercurio F (2007) Interest rate models - theory and practice: with smile, inflation and credit. Springer, Berlin

3. Cox JC, Ingersoll JE Jr, Ross SA (1985) An intertemporal general equilibrium model of asset prices. Econom J Econom Soc 30:363-384

4. Cox SH, Pedersen HW (1999) Nonparameteric estimation of interest rate term structure and insurance applications. In: Proceedings of the 1999 ASTIN Colloquium, Tokyo, Japan (to appear)

5. Dai Q, Singleton Kenneth J (2000) Specification analysis of affine term structure models. J Financ 55(5):1943-1978

6. Diebold FX, Li C (2006) Forecasting the term structure of government bond yields. J Econom 130(2):337-364

7. Diez F, Korn R (2019) Yield curve shapes of vasicek interest rate models, measure transformations and an application for the simulation of pension products. Eur Actuar J 20:1-30

8. Duffee GR (2002) Term premia and interest rate forecasts in affine models. J Financ 57(1):405-443

9. Duffie D, Kan R (1996) A yield-factor model of interest rates. Math Financ 6(4):379-406

10. Girsanov IV (1960) On transforming a certain class of stochastic processes by absolutely continuous substitution of measures. Theory Probab Appl 5(3):285-301

11. Heath D, Jarrow R, Morton A (1992) Bond pricing and the term structure of interest rates: a new methodology for contingent claims valuation. Econom J Econom Soc 20:77-105

12. Hull J, White A (1990) Pricing interest rate derivative securities. Rev Financ Stud 3(4):573-592

13. Hull J, Sokol A, White A (2014) Short rate joint measure models. Risk 10:59-63

14. de Jong F (2000) Time series and cross-section information in affine term-structure models. J Bus Econ Stat 18(3):300-314

15. Korn R, Wagner A (2019) Praxishandbuch Lebensversicherungsmathematik: Simulation und Klassifikation von Produktent. VVW GmbH, Karlsruhe

16. Nelder JA, Mead R (1965) A simplex method for function minimization. Comput J 7(4):308-313

17. Stanton R (1997) A nonparametric model of term structure dynamics and the market price of interest rate risk. J Financ 52(5):1973-2002

18. Vasicek O (1977) An equilibrium characterization of the term structure. J Financ Econ 5(2):177-188

Publisher's Note Springer Nature remains neutral with regard to jurisdictional claims in published maps and institutional affiliations. 